\title{
PROCEDIMENTOS PARA RECUPERAÇÃo DE Ag DE RESÍDUOS LÍQUIDOS E SÓLIDOS
}

\author{
José Albertino Bendassolli*, Glauco Arnold Tavares, Raquel de Fátima Ignoto e Alexssandra Luiza Rodrigues Molina \\ Rosseti \\ Centro de Energia Nuclear na Agricultura, Universidade de São Paulo, CP 96, 13400-970 Piracicaba - SP
}

Recebido em 19/8/02; aceito em 20/12/02

\begin{abstract}
RECOVERING PROCEDURES FOR LIQUID AND SOLID Ag RESIDUES. A simple procedure for recovering Ag, generated as residual solutions, from three different analytical methods, is presented. Based on the rate of the total $\mathrm{Ag}$ mass recovered, to the initial one in the initial residual solutions, efficiency as high as $97,9 \pm 2,9 \%$ was obtained in the process. The purity of Ag, as $\mathrm{Ag}_{2} \mathrm{O}$, was verified by employing this reagent in the determination of $\mathrm{S}$ in plant tissue. This leads to the generation of a solid metallic $\mathrm{Ag}$ as waste. In this situation, an $88,7 \pm 0,6 \% \mathrm{Ag}$ recovery was acquired, when a $\mathrm{HNO}_{3}$ solution was employed as solvent.
\end{abstract}

Keywords: Ag recovery; wastes; residue treatment.

\section{INTRODUÇÃO}

A prata é um elemento traço de ocorrência natural, que é muito empregado em indústrias de fotografia e imagem, bem como em eletro-eletrônicos de um modo geral. Essa acentuada utilização implica na descarga desse metal para o ambiente, o que representa risco para organismos aquáticos e terrestres ${ }^{1}$. Essa preocupação se justifica pelo seu reconhecido potencial tóxico quando despejada sem critérios no ambiente ${ }^{2-3}$.

Vários trabalhos encontrados na literatura abordam os problemas ambientais causados pela $\mathrm{Ag}$ em diversos rios no mundo, relatando contaminação de origem antropogênica em sedimentos, água e organismos aquáticos ${ }^{4-10}$.

Deixando de lado os citados danos ambientais, o despejo de Ag na forma de resíduo representa também um prejuízo financeiro, uma vez que esse metal possui significativo valor agregado. Deve-se ainda mencionar que a prata é um dos exemplos de metais com risco de escassez, recebendo inclusive atenção dos órgãos de comunicação ${ }^{11-12}$.

É importante salientar que as indústrias não são as únicas responsáveis pelo quadro atual de contaminação do ambiente por substâncias tóxicas. As universidades, escolas e institutos de pesquisa respondem por aproximadamente $1 \%$ da produção de resíduos em países desenvolvidos como os Estados Unidos ${ }^{13}$. Considerando que estas instituições exercem papel fundamental quando avaliam os impactos ambientais provocados por outras unidades geradoras de resíduos, é necessário que tratem adequadamente seus rejeitos, a fim não verem mitigada sua credibilidade perante a sociedade e os órgãos públicos competentes ${ }^{13-16}$.

Procedimentos para a recuperação de prata em rejeitos de laboratório e em chapas radiográficas, filmes e papéis fotográficos, são apresentados na literatura ${ }^{17-20}$. Nesses procedimentos, os materiais (filmes, chapas ou papéis) são imersos em solução ácida contendo tiouréia, à temperatura ambiente. A prata e seus sais são solubilizados formando o complexo $[\mathrm{Ag}(\mathrm{tu})]^{+}$. Em seguida, eleva-se o $\mathrm{pH}$ do meio com $\mathrm{NaOH}$ (7,0-8,0), formando-se precipitado de $\mathrm{Ag}_{2} \mathrm{~S}$. Essa solução é filtrada e o precipitado calcinado para obtenção da prata metálica.

Dentro do contexto supra citado, são empregadas em rotina nos laboratórios de pesquisa do CENA/USP as seguintes metodologias

*e-mail: jab@cena.usp.br que geram como resíduo solução contendo Ag: a metodologia clássica de análise de $\mathrm{DQO}^{21}$, comumente empregada em laboratórios de limnologia; um método de análise de proteínas em plantas ${ }^{22}$ e uma outra metodologia de determinação de sulfeto volátil ácido (SVA) em sedimentos ${ }^{23}$. No trabalho, foi ainda avaliada a reciclagem de prata proveniente do processo de oxidação de S-orgânico a S-Sulfato na determinação do teor de $\mathrm{S}$ e $\%$ em átomos de ${ }^{34} \mathrm{~S}$ em amostras vegetais ${ }^{24}$.

No estabelecimento do Programa de Gerenciamento de Resíduos Químicos do CENA/USP (PGRQ - CENA/USP), a recuperação da prata contida nos resíduos provenientes dos laboratórios é de grande interesse. No trabalho, a prata é recuperada na forma de $\mathrm{Ag}_{2} \mathrm{O}$ e sua reutilização foi avaliada no próprio método de oxidação de $\mathrm{S}$ orgânico a S-SO ${ }_{4}^{-2}$, objetivando a determinação de enxofre em amostras de solos e plantas ${ }^{24}$.

\section{MATERIAIS E MÉTODOS}

\section{Resíduos líquidos contendo Ag}

Nesta etapa, foi avaliada a eficiência do processo de recuperação da Ag contida nos resíduos aquosos das análises de DQO, SVA e proteínas.

Os ensaios, realizados em triplicatas, objetivando a recuperação da prata contida nas soluções residuais, foram realizados em volumes de $1000 \mathrm{~mL}$. A Figura 1 apresenta o fluxograma do processo, sendo a prata recuperada na forma de $\mathrm{Ag}_{2} \mathrm{O}$. No procedimento adotado, utilizou-se como agente precipitante da prata solução saturada de $\mathrm{NaCl}$ (200 $\left.\mathrm{g} \mathrm{L}^{-1}\right)$, ocorrendo as reações (1) e (2).

$$
2 \mathrm{NaCl}(\mathrm{aq})+1 \mathrm{Ag}_{2} \mathrm{SO}_{4}(\mathrm{aq}) \rightarrow 2 \mathrm{AgCl}(\mathrm{s})+\mathrm{Na}_{2} \mathrm{SO}_{4}(\mathrm{aq})
$$$$
\text { (resíduo de DQO) }
$$

$\mathrm{NaCl}(\mathrm{aq})+\mathrm{AgNO}_{3}(\mathrm{aq}) \rightarrow \mathrm{AgCl}(\mathrm{s})+\mathrm{NaNO}_{3}(\mathrm{aq})$

(resíduo de análises de proteínas e SAV)

onde (aq) e (s) representam as fases aquosa e sólida, respectivamente.

A baixa solubilidade do $\mathrm{AgCl}\left(\mathrm{Kps}=1,8.10^{-10}\right)$ favorece a precipitação desse composto, permanecendo na fração sobrenadante outras impurezas, como o $\mathrm{Hg}^{+2}$ (determinação de DQO), cuja solubili- 


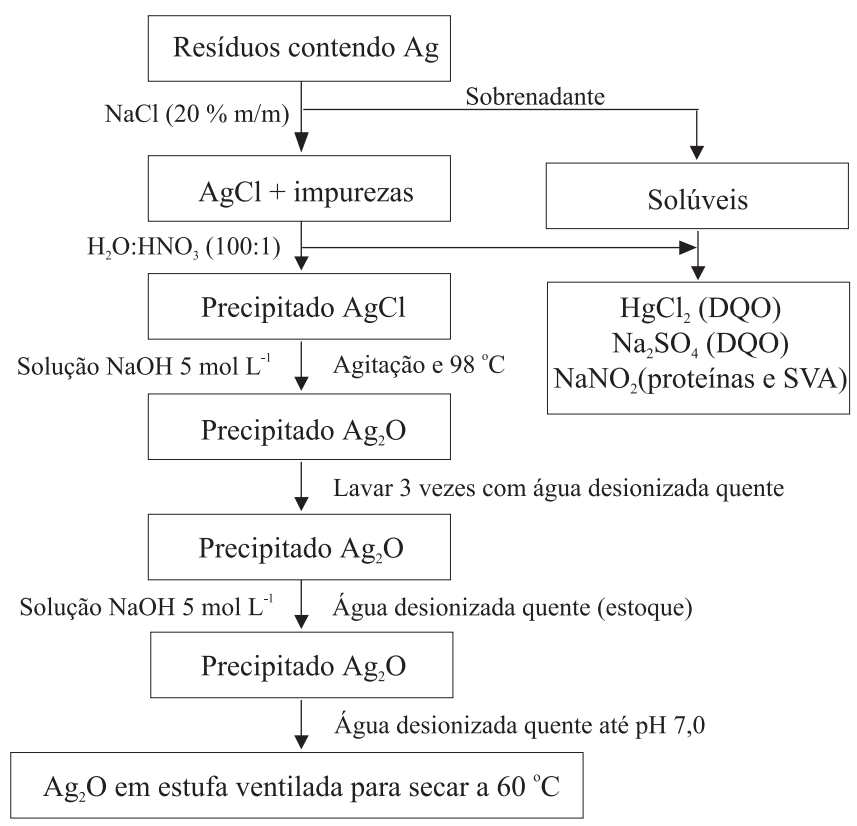

Figura 1. Fluxograma para obtenção de $\mathrm{Ag}_{2} \mathrm{O}$ a partir da solução residual contendo Ag

dade elevada ( $69 \mathrm{~g} \mathrm{~L}^{-1}$ de $\mathrm{HgCl}_{2}$ ) excede em cerca de 40 vezes a sua concentração teórica na solução residual ${ }^{25}$. Deve-se ressaltar, entretanto, que a adição de excesso de $\mathrm{NaCl}$ pode solubilizar o precipitado, devido à formação de complexos entre o cloro e a prata $\left(\mathrm{AgCl}_{2}^{-}\right.$, $\left.\mathrm{AgCl}_{3}^{-2}, \mathrm{AgCl}_{4}^{-3}\right)$. Outros compostos presentes na fração líquida residual gerada nesse procedimento são: $\mathrm{NaNO}_{3}, \mathrm{Na}_{2} \mathrm{SO}_{4}$ e formaldeído, este último gerado na metodologia de análise de proteínas.

$\mathrm{Na}$ separação quantitativa do precipitado de $\mathrm{AgCl}$ empregou-se centrifuga Beckman modelo J2-HS e, após essa etapa, o precipitado foi separado (separação do sobrenadante) e lavado com água e ácido nítrico na proporção de 100:1 para remoção de possíveis impurezas que poderiam estar adsorvidas nas partículas de $\mathrm{AgCl}$. A separação do precipitado também pode ser realizada após repouso de 24 h, procedendo-se na seqüência a separação da fase líquida. Ressalta-se que a solução de lavagem foi estocada para ser posteriormente introduzida em outro volume residual a ser processado.

Ao precipitado de $\mathrm{AgCl}$, adicionou-se solução de $\mathrm{NaOH} 5 \mathrm{~mol} \mathrm{~L}^{-1}$, promovendo-se a reação (3)

$2 \mathrm{AgCl}(\mathrm{s})+2 \mathrm{NaOH}(\mathrm{aq}) \rightarrow \mathrm{Ag}_{2} \mathrm{O}(\mathrm{s})+2 \mathrm{NaCl}(\mathrm{aq})+1 \mathrm{H}_{2} \mathrm{O}$

A adição da base foi realizada em sistema com constante agitação e aquecimento até a ebulição. Após essa etapa, efetuaram-se lavagens sucessivas com água desionizada, seguidas de centrifugação, sendo as soluções sobrenadantes (contendo $\mathrm{NaOH}$ ) acondicionadas para posterior utilização em outros procedimentos de tratamento de resíduos (por exemplo, precipitação de metais pesados ou neutralização de soluções residuais ácidas).

Para se garantir a eficiência do processo, efetuou-se nova adição de $\mathrm{NaOH}$ e as subsequientes etapas de lavagem. Por fim, a secagem foi realizada em estufa ventilada a $60{ }^{\circ} \mathrm{C}$.

A eficiência da metodologia proposta foi avaliada, primeiramente, comparando-se a massa de $\mathrm{Ag}_{2} \mathrm{O}$ produzida em relação à massa teórica possível de se obter. Em seguida, a qualidade do $\mathrm{Ag}_{2} \mathrm{O}$ obtido foi aferida empregando-se esse composto recuperado e $\mathrm{Ag}_{2} \mathrm{O}$ Sigma p.a. na determinação isotópica de ${ }^{34} \mathrm{~S}$ em amostras vegetais, na qual esse reagente é empregado para conversão de S-orgânico para Ssulfato $^{24-26}$. A validação foi realizada empregando-se amostras vegetais padrão (amostras do Programa Interlaboratorial de Análise de Tecido Vegetal, Ano 12 (biênio 1996/1997), Departamento de Solos e Nutrição de Plantas, ESALQ/USP), aplicando-se o teste t pareado à nível de significância de $1 \%$ de probabilidade.

\section{Resíduos sólidos contendo Ag}

Nesta etapa, foi avaliada a eficiência do processo de recuperação de Ag contida nos resíduos sólidos, proveniente do processo de conversão de S-orgânico a S-Sulfato em amostras vegetais, utilizando-se do método por via seca ${ }^{24,27}$. Nesse método, os reagentes $\mathrm{Ag}_{2} \mathrm{O}$ e $\mathrm{NaHCO}_{3}$ (razão $1: 10 \mathrm{~m} / \mathrm{m}$ ), juntamente com a amostra vegetal de interesse (0,5 a 1,0 g MS), são acondicionados em um cadinho de porcelana e levados à mufla por $8 \mathrm{~h}$, à temperatura de $550^{\circ} \mathrm{C}$. Ao final do processo obtêm-se prata na forma metálica, juntamente com outros resíduos sólidos (cinzas e outros). Na massa sólida resultante adicionam-se cerca de $50 \mathrm{~mL}$ de água desionizada e o volume é submetido a agitação por 15 min (solubilização do sulfato). Na seqüência, efetuase a filtragem utilizando-se papel de filtro de fibra de vidro de $0,45 \mu \mathrm{m}$. A prata metálica contida no papel de filtro é separada mecanicamente, após secagem do material (filtro + prata + resíduos sólidos). A massa final contendo resíduos sólidos é calcinada a $870{ }^{\circ} \mathrm{C}$ por um período de 4 h. Na seqüência, adiciona-se ácido nítrico $65 \%(\mathrm{~m} / \mathrm{v})$ sobre a prata obtendo-se nitrato de prata de acordo com a reação (4).

$\mathrm{Ag}^{\mathrm{o}}(\mathrm{s})+\mathrm{HNO}_{3}(\mathrm{aq}) \rightarrow \mathrm{AgNO}_{3}(\mathrm{aq})+1 / 2 \mathrm{H}_{2}(\mathrm{~g})$

onde (s), (aq) e (g) representam as fases sólida, aquosa e gasosa, respectivamente.

A reação representada na equação (4) ocorre em sistema aberto, à temperatura da ordem de $90{ }^{\circ} \mathrm{C}$, e com excesso de $50 \%$ de ácido nítrico ( $1 \mathrm{~mL}$ de $\mathrm{HNO}_{3}$ concentrado para cada grama de prata). Após concluída a reação, o volume da solução contendo $\mathrm{AgNO}_{3}$ é avolumado para $50 \mathrm{~mL}$ com adição de água desionizada. Na próxima etapa adiciona-se solução saturada de cloreto de sódio $\left(200 \mathrm{~g} \mathrm{~L}^{-1}\right.$ de $\mathrm{NaCl}$ ), com excesso de $50 \%$, de acordo com a estequiometria da reação (5).

$\mathrm{AgNO}_{3}(\mathrm{aq})+\mathrm{NaCl}(\mathrm{aq}) \rightarrow \mathrm{AgCl}(\mathrm{s})+\mathrm{NaNO}_{3}(\mathrm{aq})$

Na etapa seguinte procede-se a separação e lavagem da fase sólida $(\mathrm{AgCl})$, utilizando-se sistema de trompa de vácuo e água desionizada (duas ou três vezes). Finalmente adiciona-se solução de hidróxido de sódio $18 \mathrm{~mol} \mathrm{~L}^{-1}$ sobre o $\mathrm{AgCl}$, resultando na formação do $\mathrm{Ag}_{2} \mathrm{O}$ de acordo com a reação (3). Com a realização dos procedimentos descritos anteriormente determina-se a massa de $\mathrm{Ag}_{2} \mathrm{O}$ obtida e calcula-se o rendimento do processo. A reutilização do $\mathrm{Ag}_{2} \mathrm{O}$, assim obtido, pode então ser verificada empregando esse reagente e $\mathrm{Ag}_{2} \mathrm{O}$ p.a (Sigma) na conversão de S-Orgânico (amostras vegetais) a S-SO ${ }_{4}^{-2}$.

\section{RESULTADOS E DISCUSSÃO}

\section{Resíduos líquidos contendo Ag}

A eficiência determinada em testes realizados no CENA/USP comprovou a viabilidade do tratamento proposto nos resíduos líquidos contendo prata. A recuperação da prata (na forma de $\mathrm{Ag}_{2} \mathrm{O}$ ) inicialmente contida na solução residual teórica $\left(1,08 \mathrm{~g} \mathrm{~L}^{-1}\right.$ de solução residual de DQO; $1,25 \mathrm{~g} \mathrm{~L}^{-1}$ de solução residual de análise de proteínas e $2,87 \mathrm{~g} \mathrm{~L}^{-1}$ de solução residual de SVA), em função do volume processado, pode ser observada na Tabela 1 . 
Tabela 1. Recuperação da prata, na forma de $\operatorname{Ag}_{2} \mathrm{O}$, proveniente das soluções residuais geradas nos três procedimentos analíticos (média de 3 repetições)

\begin{tabular}{cccc}
\hline Solução residual & Massa Teórica* $\mathrm{Ag}_{2} \mathrm{O}(\mathrm{g})$ & Massa Obtida $\mathrm{Ag}_{2} \mathrm{O}(\mathrm{g})$ & Rendimento $(\%)$ \\
\hline DQO & 1,08 & $1,12 \pm 0,06$ & $103,7 \pm 5,6$ \\
Análise de proteínas & 1,25 & $1,20 \pm 0,03$ & $96,0 \pm 2,5$ \\
SVA & 2,87 & $2,70 \pm 0,07$ & $94,1 \pm 2,6$ \\
Média & & & $97,9 \pm 2,9$ \\
\hline
\end{tabular}

* valor obtido a partir da estequiometria dos métodos analíticos utilizados

Após a determinação gravimétrica do rendimento, empregou-se a Ag recuperada na forma de $\mathrm{Ag}_{2} \mathrm{O}$ para a determinação elementar (\% de $\mathrm{S}$ na amostra) e isotópica (\% átomos de ${ }^{34} \mathrm{~S}$ ) em amostras vegetais padrão (amostras do Programa Interlaboratorial de Análise de Tecido Vegetal - ano 12 - Escola Superior de Agricultura "Luiz de Queiroz”, Departamento de Solos e Nutrição de Plantas). Os resultados relacionados com o teor de $\mathrm{S}$ nas amostras $\left(\mathrm{g} \mathrm{kg}^{-1}\right.$ de $\left.\mathrm{S}\right)$, utilizando-se $\mathrm{Ag}_{2} \mathrm{O}$ obtido das soluções residuais de DQO, foram comparados com aqueles onde se empregou o $\mathrm{Ag}_{2} \mathrm{O}$ Sigma p.a.. A Tabela 2 apresenta esses dados, bem como os testes estatísticos (teste $\mathrm{T}$ pareado de Student). A Tabela 2 contém ainda os resultados médios da concentração de $\mathrm{S}$ nas amostras avaliadas dentro do programa interlaboratorial (56 laboratórios).

As determinações do teor de $\mathrm{S}$ empregando $\mathrm{Ag}_{2} \mathrm{O}$ recuperado proporcionaram resultados em conformidade estatística com os valores de referência, sendo ainda mais próximos destes que quando comparado ao reagente Sigma p.a. (Tabela 2).

Os resultados obtidos caracterizaram a viabilidade do emprego dessa prática em atividades de rotina, proporcionando vantagens em termos ambientais e econômicos. Vale ressaltar que o custo aproximado de $\mathrm{Ag}_{2} \mathrm{O}$ Sigma p.a. é de $\mathrm{R} \$ 13,00$ o grama (aproximadamente U\$ 5,00), enquanto os regentes usados para essa recuperação, bem como os equipamentos empregados, dispensam grandes investimentos. Estima-se que os resíduos provenientes das determinações de DQO, SVA e proteínas, provenientes dos laboratórios de pesquisa do CENA/USP, totalizem 450 a $500 \mathrm{~g}$ de $\mathrm{Ag}$ anualmente. Esse material, na forma de $\mathrm{Ag}_{2} \mathrm{O}$, pode ser avaliado em aproximadamente $\mathrm{R} \$ 6.500,00$, e pode ser recuperado, a partir do resíduo inicial, com custo muito baixo (cerca de $\mathrm{R} \$ 200,00$ ).
Além da recuperação da prata, a solução residual da determinação de DQO ainda contém outros metais pesados (principalmente mercúrio e cromo), presentes na solução sobrenadante resultante da primeira etapa de precipitação (reação com $\mathrm{NaCl}$ ), descrita no fluxograma apresentado na Figura 1. Embora não seja intenção detalhar também os procedimentos adotados para o tratamento dessa solução, o princípio baseou-se na precipitação dos metais pesados nas formas de hidróxidos e/ou sulfetos, resultando em resíduos sólidos a serem temporariamente estocados em depósito apropriado. Com relação ao $\mathrm{Cr}(\mathrm{VI})$, deve-se inicialmente promover a sua redução para Cr(III), empregando tiossulfato de sódio ou metabissulfito de sódio em pH ácido, para posterior precipitação do $\mathrm{Cr}(\mathrm{OH})_{3}$ e filtragem ${ }^{28}$. Esses procedimentos foram repetidos até que a solução resultante contivesse concentrações de $\mathrm{Hg}$ e $\mathrm{Cr}$ inferiores a $10 \mu \mathrm{g} \mathrm{L}^{-1}$ e $5 \mathrm{mg} \mathrm{L}^{-1}$, respectivamente, podendo ser descartada na forma de efluente em um rio de classe $2^{29}$.

Conclui-se por fim que, através da adoção de procedimentos dessa natureza, se pode contribuir para a minimização de problemas ambientais, para a formação de recursos humanos acostumados às práticas de tratamento de resíduos (no caso de universidades, escolas e institutos de pesquisa) e, no caso da recuperação da prata, possibilitar retorno financeiro.

\section{Resíduos sólidos contendo Ag}

A recuperação da $\mathrm{Ag}$ proveniente do processo de conversão de S-orgânico a S-Sulfato em amostras vegetais foi avaliada em resíduos com diferentes massas do metal. Desta forma, foi possível verificar a eficiência ou rendimento do processo em resíduo contendo

Tabela 2. Concentração de $\mathrm{S}$ ( $\mathrm{g} \mathrm{kg}^{-1}$ de $\mathrm{S}$ ) em amostras vegetais do Programa Interlaboratorial (valor médio de 56 laboratórios), empregandose $\mathrm{Ag}_{2} \mathrm{O}$ recuperado e Sigma p.a. (3 repetições)

\begin{tabular}{|c|c|c|c|}
\hline Amostra & $\mathrm{Ag}_{2} \mathrm{O}$ recuperado (a) $\left(\mathrm{g} \mathrm{kg}^{-1}\right.$ de $\left.\mathrm{S}\right)$ & $\mathrm{Ag}_{2} \mathrm{O}$ Sigma p.a. (b) $\left(\mathrm{g} \mathrm{kg}^{-1}\right.$ de $\left.\mathrm{S}\right)$ & Valor de referência ${ }^{(c)}\left(\mathrm{g} \mathrm{kg}^{-1}\right.$ de $\left.S\right)$ \\
\hline CITRUS & $2,29 \pm 0,21$ & $2,06 \pm 0,19$ & $2,25 \pm 0,24$ \\
\hline CAFÉ & $1,89 \pm, 16$ & $1,80 \pm 0,16$ & $1,92 \pm 0,22$ \\
\hline INT 02 & $1,56 \pm 0,14$ & $1,51 \pm 0,15$ & $1,44 \pm 0,13$ \\
\hline INT 04 & $1,80 \pm 0,17$ & $1,79 \pm 0,16$ & $1,95 \pm 0,21$ \\
\hline INT 08 & $2,59 \pm 0,19$ & $2,58 \pm 0,22$ & $2,61 \pm 0,23$ \\
\hline INT 09 & $7,36 \pm 0,76$ & $7,30 \pm 0,82$ & $7,37 \pm 1,30$ \\
\hline INT 13 & $1,52 \pm 0,13$ & $1,50 \pm 0,13$ & $1,85 \pm 0,25$ \\
\hline INT 14 & $3,56 \pm 0,37$ & $3,50 \pm 0,16$ & $4,24 \pm 0,54$ \\
\hline INT 15 & $2,60 \pm 0,22$ & $2,55 \pm 0,23$ & $2,76 \pm 0,26$ \\
\hline INT 08 & $1,90 \pm 0,18$ & $1,88 \pm 0,18$ & $2,22 \pm 0,19$ \\
\hline Teste $\mathrm{t}$ pareado* & $2,06 \mathrm{~ns}^{* *}$ & $2,36 \mathrm{~ns}$ & \\
\hline
\end{tabular}

(a) determinação do teor de $\mathrm{S}$ (amostras orgânicas) utilizando-se $\mathrm{Ag}_{2} \mathrm{O}$ recuperado do resíduo; (b) determinação do teor de $\mathrm{S}$ (amostras orgânicas) utilizando-se $\mathrm{Ag}_{2} \mathrm{O}$ sigma P.A.; ${ }^{(c)}$ valor de referência do teor de S referente aos 56 laboratórios participantes do programa interlaboratorial. *teste $\mathrm{t}$ referente aos valores de teor de $\mathrm{S}$ nas amostras do programa de intercalibração; **valor de t seguido de ns representa não significativo no nível de significância de $1 \%$ de probabilidade 
Tabela 3. Rendimento do processo de reciclagem da prata proveniente de resíduos sólidos do processo de combustão de S-orgânico

\begin{tabular}{cccc}
\hline Massa de prata residual $(\mathrm{g})$ & Massa de $\mathrm{Ag}_{2} \mathrm{O}$ obtida $(\mathrm{g})$ & Massa de prata recuperada $(\mathrm{g})$ & Rendimento do processo $(\%) *$ \\
\hline 1,93 & 1,86 & 1,73 & 89,6 \\
8,11 & 7,92 & 7,38 & 91,0 \\
1,97 & 1,83 & 1,71 & 86,8 \\
2,49 & 2,34 & 2,18 & 87,6 \\
3,83 & 3,63 & 3,38 & 88,3 \\
1,09 & 1,04 & 0,97 & 89,0 \\
& & & Média $\pm \mathrm{sd} * *, 7 \pm 0,6$
\end{tabular}

$*$ Rendimento $=($ massa de prata recuperada/massa de prata residual $) .10^{2} ; * * \mathrm{sd}=$ desvio padrão da média

de 1,09 a 8,11 g de Ag na forma metálica. No Laboratório de Isótopos Estáveis (CENA/USP) estima-se que $100 \mathrm{~g}$ de Ag podem ser recuperadas anualmente com o processo descrito no trabalho $(\mathrm{R} \$ 1.300,00)$.

A Tabela 3 apresenta os dados obtidos para seis ensaios realizados, a partir de resíduos sólidos provenientes das determinações isotópicas (\% em átomos de ${ }^{34} \mathrm{~S}$ ) e teor de $\mathrm{S}(\% \mathrm{~S})$ em amostras vegetais. Esses dados mostram que o rendimento do processo de reciclagem de $\mathrm{Ag}$ da forma metálica para $\mathrm{Ag}_{2} \mathrm{O}$ é, em média, de 88,7 $\pm 0,6 \%$. $\mathrm{O} \mathrm{Ag}_{2} \mathrm{O}$ obtido com o procedimento pode ser novamente utilizado na etapa de oxidação do S-orgânico (enxofre combinado) a $\mathrm{S}_{-} \mathrm{SO}_{4}{ }^{-2}$, em substituição ao produto grau analítico. Deve-se ainda destacar que o processo é viável economicamente e possibilita a reciclagem deste importante metal.

\section{CONCLUSÕES}

Os resultados experimentais indicam que é possível recuperar anualmente cerca de $500 \mathrm{~g}$ de $\mathrm{Ag}$ a partir de resíduos aquosos proveniente das análises de DQO, SVA e proteínas, e outras $100 \mathrm{~g}$ do metal a partir de resíduos sólidos gerados no processo de determinação de $\mathrm{S}(\%)$ e ${ }^{34} \mathrm{~S}$ (\% átomos de ${ }^{34} \mathrm{~S}$ ) em amostras vegetais. A prata recuperada, na forma final de $\mathrm{Ag}_{2} \mathrm{O}$, pode ser utilizada no processo de determinação de $\mathrm{S}(\%)$ e ${ }^{34} \mathrm{~S}$ (\% átomos) por via seca, levando-se em consideração os resultados obtidos no programa de intercalibração laboratorial. O método utilizado possibilita a recuperação aproximada de 98 e $89 \%$ da prata contida nos resíduos aquosos e sólidos, respectivamente. $\mathrm{O}$ processo apresenta viabilidade econômica, sendo possível recuperar cerca de $\mathrm{R} \$ 7.800,00$ anualmente a partir dos resíduos provenientes dos laboratórios de pesquisa do CENA/USP.

\section{AGRADECIMENTOS}

Os autores agradecem à FAPESP, ao CNPq e CAPES pelos auxílios financeiros e bolsas concedidas.

\section{REFERÊNCIAS}

1. Purcell, T. W.; Peters, J. J.; Environ. Toxicol. Chem. 1998, 17, 539.
2. Gorsuch, J. W.; Klaine, S. J.; Environ. Toxicol. Chem. 1998, 17, 537.

3. Lima, A. R.; Curtis, C.; Hammermeister, D. E.; Call, D. J.; Felhaber, T. A.; Bull. Environ. Contam. Toxicol. 1982, 29, 184.

4. Wen, L. S.; Santschi, P. H.; Gill, G. A.; Paternostro, C. L.; Lehman, R. D.; Environ. Sci. Technol. 1997, 31, 723.

5. Gobeil, C.; Environ. Sci. Technol. 1999, 33, 253.

6. Yamazaki, M.; Tanizaki, Y.; Shimokawa, T.; Environ. Pollut. 1996, 94, 83.

7. Riedel, G. F.; Abbe, G. R.; Sanders, J. G.; Estuaries 1995, 18, 445.

8. Whitlow, S. I.; Rice, D. L.; Water Res. 1985, 19, 619.

9. Ramelow, G. J.; Webre, C. L.; Mueller, C. S.; Beck, J. N.; Young, J. C.; Langley, M. C.; Arch. Environ. Contam. Toxicol. 1989, 18, 804.

10. Nakamoto, R. J.; Hassler, T. J.; Arch. Environ. Contam. Toxicol. 1992, 22, 88.

11. Fellenberg, G.; Introdução aos problemas da poluição ambiental, EDUSP: São Paulo, 1980.

12. Skinner, B. J.; Recursos minerais da Terra, Edgard Blucher: São Paulo, 1970.

13. Ashbrook, P. C.; Reinhardt, P. A.; Environ. Sci. Technol. 1985, 19, 1150.

14. Jardim, W. F.; Quim. Nova 1998, 21, 671.

15. Kaufman, J. A. Em Waste disposal in academic instituitions; Kaufman, J. A., ed.; Lewis: Michigan, 1990, p. 93.

16. Schneider, J.; Wiskamp, V.; J. Chem. Educ. 1994, 71, 587.

17. Oliveira, D.; Uchinei, F. Y.; Bezerra, S.; Métodos de recuperação e reciclagem de prata de rejeitos de laboratório, S N: São Paulo, 1983.

18. Sobral, L. G. S.; Granato, M.; Recuperação de prata de fixadores fotográficos, Série Tecnologia Mineral, Dnpm: Brasília, 1984.

19. Ayata, S.; Yildiran, H.; Turk. J. Chem. 2001, 25, 187.

20. Martins, E. A. J.; Abrão, A.; Resumos do $1^{\circ}$ Encontro Nacional de Segurança em Química, Campinas, Brasil, 2000.

21. Golterman, H. L.; Clymo, R. S.; Ohnstad, M. A. M.; Methods for physical and chemical analysis of fresh water, $2^{\text {th }}$ ed., Blackwell: Oxford, 1978.

22. Blum, H.; Beier, H.; Gross, H. J.; Electrophoresis 1987, 8, 93.

23. Di Toro, D. M.; Mahony, J. D.; Hansen, D. J.; Scott, K. J.; Hicks, M. B.; Mayr, S. M.; Redmond, M. S.; Environ. Toxicol. Chem. 1990, 9, 1487.

24. Carneiro Jr, F.; Tese de Doutorado, Universidade de São Paulo, Brasil, 1998.

25. Quagliano, J. V.; Vallarino, L. M.; Química, Guanabara: Rio de Janeiro, 1973.

26. Tabatabai, M. A.; Methods of Soil Analysis, American Society of Agronomy: Madison, 1982.

27. Schoenau, J.; Bettany, J. R.; Soil Sci. Soc. Am. J. 1988, 52, 297.

28. Armour, M.; J. Chem. Educ. 1988, 65, 64.

29. Companhia de Tecnologia de Saneamento Ambiental; Guia de coleta e preservação de amostras de água, CETESB: São Paulo, 1987. 